\title{
MEASUREMENT OF THE THRESHOLD SENSITIVITY OF HONEYBEES TO WEAK, EXTREMELY LOW-FREQUENCY MAGNETIC FIELDS
}

\author{
JOSEPH L. KIRSCHVINK*, S. PADMANABHA, C. K. BOYCE AND J. OGLESBY \\ Division of Geological and Planetary Sciences, The California Institute of Technology 170-25, Pasadena, \\ CA 91125, USA
}

Accepted 24 February 1997

\begin{abstract}
Summary
Experiments reported previously demonstrate that freeflying honeybees are able to detect static intensity fluctuations as weak as $26 \mathrm{nT}$ against the background, earth-strength magnetic field. We report here an extension

increasing frequency. At $60 \mathrm{~Hz}$, alternating field strengths above $100 \mu \mathrm{T}$ are required to elicit discrimination. These results are consistent with biophysical predictions of a magnetite-based magnetoreceptor.
\end{abstract} of this work to weak, alternating fields at frequencies of $\mathbf{1 0}$ and $60 \mathrm{~Hz}$. Our results indicate that the sensitivity of the honeybee magnetoreception system decreases rapidly with
Key words: magnetoreception, honeybee, Apis mellifera carnica, biogenic magnetite, threshold sensitivity.

\section{Introduction}

During the past decade, it has been shown repeatedly that free-flying honeybees can be trained in discrimination experiments to respond to local magnetic anomalies (Walker and Bitterman, 1985, 1989a,b; Kirschvink and KobayashiKirschvink, 1991; Kirschvink et al. 1992). Honeybees are therefore one of the best experimental animals available for testing biophysical hypotheses concerning the sensory transduction of the geomagnetic field in animals. In particular, Walker and Bitterman (1989b) developed an experimental protocol in which individual honeybees were trained to discriminate between the presence or absence of a small static (d.c.) magnetic anomaly in one of two targets mounted vertically. Kirschvink et al. (1992) reported the replication of this paradigm and used it to measure the range of frequencies, of constant amplitude, to which the honeybee magnetoreceptor system was capable of responding; they demonstrated an ability to detect alternating (a.c.) fields of $2.2 \mathrm{mT}$ peak amplitude from d.c. at frequencies up to $60 \mathrm{~Hz}$. Above $60 \mathrm{~Hz}$, the behavior approached randomness. This result was broadly consistent with the biophysical prediction for a magnetitebased magnetoreceptor (Kirschvink et al. 1992).

We report here an extension of these experiments in which the modified Walker and Bitterman (1989b) protocol was used to place relative constraints on the threshold sensitivity of honeybees to a.c. magnetic fields at frequencies of 10 and $60 \mathrm{~Hz}$, complementing their results for static magnetic anomalies. We find that the magnetic sense of the honeybee has its maximum sensitivity below $10 \mathrm{~Hz}$, which is consistent with the evolution of this system within the known geomagnetic frequency spectrum and with a viscously damped ferromagnetic magnetoreceptor.

\section{Materials and methods}

Fig. 1 shows one of the two identical targets used in the experiments, which were modeled after those used by Walker and Bitterman (1989b). A focused magnetic anomaly is generated locally at the target area by two sets of coils. The outer coil has a $5 \mathrm{~cm}$ radius and a total of eight turns, whereas the inner coil has a $2 \mathrm{~cm}$ radius and 50 turns. Because the current multiplied by area for these coils is equal $(5 \times 5 \times 8=2 \times 2 \times 50)$, these coils have equal dipole moments but, as they are wired in opposition, their fields cancel at large distances from the source. Numerical modeling of the field from this configuration and direct field measurements demonstrate that the magnetic flux produced by the inner coil is confined closely to the area surrounding the target, with virtually no residual field outside the area of the outer coil (Kirschvink and Kobayashi-Kirschvink, 1991). As in the Walker and Bitterman (1989b) experiments, each coil is double-wrapped so that, if the current is flowing in the same direction, the magnetic field will be generated at the center; if it is in the antiparallel mode, the magnetic field from each wire pair will cancel, yielding no external field but producing similar ohmic heating and vibrational artifacts. The wires were cemented in place as they were wound to eliminate magnetomechanical motion between them produced by our use of a.c. fields. Further details are given elsewhere (Kirschvink, 1992; Kirschvink et al. 1992).

\footnotetext{
*e-mail:krschvnk@ caltech.edu.
} 


\section{J. L. KIRSCHVINK AND OTHERS}

Fig. 1. Schematic diagram of the experimental assembly, modified from that of Walker and Bitterman (1989b). Two identical assemblies are mounted on a north-facing vertical window, separated by $15 \mathrm{~cm}$. Within each assembly, the double-wrapped coils are connected in series such that the inner and outer coils are in opposition (therefore, when activated, the dipole moments are aligned in opposite directions). This yields a sharply focused magnetic anomaly centered on the landing platform/tunnel assembly. The two target assemblies are also wired in series, but arranged such that one target is in the magnetically active mode, while the other is in the 'active-sham' mode (see Kirschvink, 1992). The outside of each target has a rectangular patch of yellow plastic over the tunnel to aid the honeybees in finding the tunnel entrance. An infrared emitter/photocell pair (IR-beam) positioned across the tunnel entrance automatically signaled the presence of a honeybee.

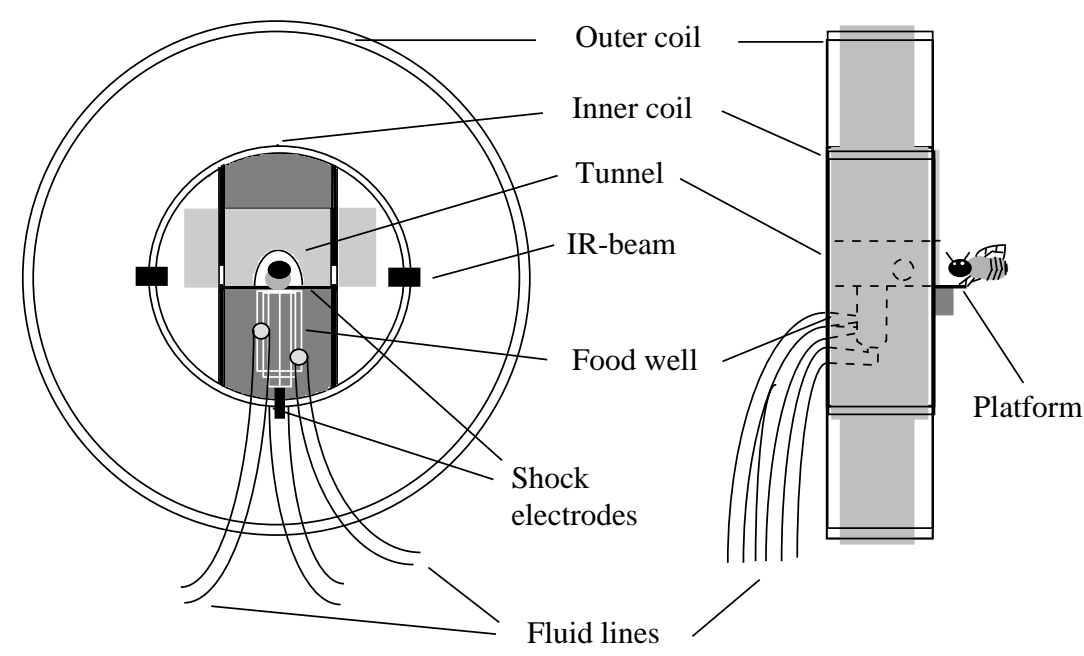

Back (inside) view
Side view
At the center of the inner coil of both target assemblies was a landing platform assembly and food well which differed slightly from that used in the Walker and Bitterman (1989b) experiment. The landing platforms were made from a coppercoated electrical circuit board, cut into a thick T-shape, with the copper foil back-plated with a thin layer of tin (Sn) to prevent corrosion of the conductive surface by Los Angeles smog. This $\mathrm{Cu}-\mathrm{Sn}$ surface was normally short-circuited via a small relay to a fine gold wire at the base of the food well (except, as noted below, when a mild shock was delivered). A matched infrared emitter/photocell pair was positioned across the entrance to the target area such that a bee would be forced to break the beam (signaling its presence to the controlling computer) before being able to reach the food well. The infrared emitters and detectors were located $2 \mathrm{~cm}$ away from the center of the assembly, as all models we tested contained a small but measurable amount of a ferromagnetic material. After demagnetization and relocation to a distance of $2 \mathrm{~cm}$ from the center of the assembly, the static magnetic field from this source at the tunnel was less than $1 \mathrm{nT}$. Unlike the experiment of Walker and Bitterman (1989b), interruption of the infrared beam was our only measure of the response of an animal. Walker and Bitterman (1989b) also used an electrical resistance detector to determine whether the bee was making contact between the landing platform and the liquid, but, as noted below, we could not use this alternative response signal as no liquid is present initially in the food wells in our revised protocol.

The food well assembly was machined from a block of Teflon mounted directly beneath the base of the T-shaped landing platform described above. A vertical hole $9.9 \mathrm{~mm}$ in diameter was drilled through the platform and into the Teflon block as shown on Fig. 1, leaving a V-shaped bottom. A drain hole was installed at the base, machined so that a $7.8 \mathrm{~mm}$ (i.d.) polyurethane connector could be inserted snugly. Two slightly smaller holes $(4.3 \mathrm{~mm}$ i.d.) were drilled into the back of the feeding well, a lower one used to deliver $50 \%$ sucrose solution, and an upper one for delivery of Pasadena tap water. It is important for the water inlet to be above that for the sugar, so that it can wash away any sucrose residue during the cleaning cycle after each visit. Fluid levels for both the $50 \%$ sucrose solution and tap water were regulated using computeractivated solenoid valves, and a gravity feeder system was used to give gentle positive pressure to each supply line. A similar solenoid valve connected to a vacuum system was used to drain the feeding well. Six computer-controlled solenoid valves were needed for this experiment, one each for the sucrose, water and drain on both target assemblies. This system allowed the computer to fill the food well partially with either water or sucrose and to rinse and drain them after each use.

With this automated delivery system, we were able to eliminate an undesirable feature of the original Walker and Bitterman (1989b) experiment, which was that the two feeders were not precisely identical when the animals were making their choice. Walker and Bitterman (1989b) used a manual system, where pipettes were used to fill the reinforced $(\mathrm{S}+)$ target with $50 \%$ sucrose solution and the punished (S-) target with tap water prior to each visit. If the honeybee was able to smell the difference, or somehow gain a subtle cue from the experimenter watching its behavior, it might confound the results [although this is unlikely in the Walker and Bitterman (1989b) experiment, given the failure of the honeybees to discriminate at low field levels]. In our experiment, each target remains empty until after the honeybee makes her choice by interrupting the infrared beam. If her first choice is correct, the food well fills rapidly with sucrose solution and the animal feeds to repletion before returning to the hive. An incorrect choice, however, is punished by the immediate introduction of tap water and a mild $(4.5 \mathrm{~V})$ electric shock, administered between the water and the metallic surface of the landing platform. As in the Walker and Bitterman (1989b) experiment, the honeybee must enter the correct target to obtain the sucrose 
solution, and can feed to repletion before returning to the hive. In our experiment, no information is available to the honeybee or to the observers in the room concerning which targets are programmed to be $\mathrm{S}+$ or $\mathrm{S}-$ until after the initial choice is made. The only exception is a single letter (E or $\mathrm{W}$ ) on a dimly lit computer screen in a darkened room approximately $3 \mathrm{~m}$ from the targets; this is clearly invisible to the honeybee and not normally monitored by the experimenter, except during occasional checking of the system operation.

In other respects, we followed the Walker and Bitterman (1989b) protocol closely. Two hives of the Italian honeybee [Apis mellifera carnica (Pollm.)] were located directly under a north-facing, third-floor window of the Arms Laboratory on the Caltech campus, where the targets were installed; this position avoided direct sunlight on the targets, and locating the two hives approximately $7 \mathrm{~m}$ directly below the experimental window seemed to minimize the number of interloping honeybees which were able to find the experiment. A 10-15\% sugar-water bee feeder was set up at a west-facing window on the third floor of the same building, from which several hundred honeybees fed. From this population, we kept approximately $5-10$ animals labeled distinctively with brightly colored lacquer (fingernail polish), allowing us to choose an individual with a fast return time for the experiment. This honeybee was caught upon arrival at the feeder in a small matchbox and released on a drop of $50 \%$ sucrose solution placed on one of the targets at the window. After a few such placements from the feeder to the target, the animal could usually find her own way back to the feeding targets, and we gradually moved the drop of sucrose solution into the hole until the animal was feeding directly from the well. During this initial training, the landing platform and entry to one of the targets was blocked manually by a plastic door, forcing the animal to enter the other target. If more than one placement trip from the feeder was required, the animal was alternated between targets. The target upon which the honeybee was placed was always the one with the active magnetic field. Upon the animal's first return directly to one of the targets, we started a six-visit forced training exercise as carried out by Walker and Bitterman (1989b) in which the honeybee was only allowed entry into alternate targets for sugar. The plastic door on the incorrect target was closed to prevent entry, and the open door was changed manually between visits so that it alternated between targets. The target with the active magnetic anomaly was always the open one during this phase.

After the sixth visit in this series, both target doors were opened and the discriminative training began with the strongest field level at the landing platform entrance. As in the Walker and Bitterman (1989b) experiment, the S+ target (E or W) was alternated between feeding visits in a balanced quasi-random sequence. Both food targets were empty prior to arrival on each visit. On a correct first response, the computer briefly opened the solenoid valve for the $50 \%$ sucrose solution, allowing the animal to feed. This typically took between 30 and $50 \mathrm{~s}$. If the beam was broken for longer than $20 \mathrm{~s}$ prior to the animal's departure, the computer waited for an additional $20 \mathrm{~s}$ before draining the well, rinsing both wells with water, and draining them in preparation for the next visit. At this time, a relay was set to switch the magnetic field to the target which would be $\mathrm{S}+$ for the next trial. The human experimenter was prompted by an audio tone upon arrival of the animal, to verify that the correctly labeled honeybee was at the feeder.

Our procedure for determining the weakest field level at which an animal was able to discriminate followed closely that of Walker and Bitterman (1989b). A string of six correct choices in a row, or seven out of eight (chance probabilities 0.016 and 0.035 , respectively) or better was used as the operational criterion that the animal had learned the discrimination task. After that visit, the peak current flowing through the coil system (and hence the field level) was reduced by half a $\log _{10}$ unit, and the experiment was continued to determine whether the animal could re-establish discrimination. Experimentation was discontinued if an animal failed to reach criterion performance after more than approximately 80 visits or if it failed to return. Animals were captured at the end of an experiment to avoid interference with subsequent work.

\section{Results}

Eleven honeybees were tested with a $60 \mathrm{~Hz}$ a.c. field and 15 at $10 \mathrm{~Hz}$. Fig. 2 shows partial results for an individual that discriminated a $10 \mathrm{~Hz}$ a.c. field down to the $4.3 \mu \mathrm{T}$ level. This honeybee began to discriminate the $1300 \mu \mathrm{T}$ field after visit 54 (results not shown), with a string of 10 correct choices out of 11 (chance probability 0.006). After this string, the field was reduced to $430 \mu \mathrm{T}$, until visit 80 when darkness finally prevented the animal from returning (results not shown). Following the protocol of Walker and Bitterman (1989b), the following morning (day 2), the field was increased back to the $1300 \mu \mathrm{T}$ level that the animal had discriminated successfully on day 1 , and after seven additional visits it again began a string of correct choices ( $7 / 8$, results not shown). At this point (visit 97), the field was reduced to the $430 \mu \mathrm{T}$ level. At visit 124 (shown on the upper diagram), the animal again began a criterion string (7/8), after which the field was reduced to $130 \mu \mathrm{T}$. It made one error, then again reached criterion (6/6), so the field was reduced to $43 \mu \mathrm{T}$ for visit 139 . At this point, the animal took an additional 49 visits before again reaching criterion (6/6) after visit 196 (data not shown); the field was therefore dropped to the $13 \mu \mathrm{T}$ level. It subsequently took 59 visits to reach criterion again with a string of $6 / 6$, starting at visit 255 (shown on the lower diagram). Following the protocol, we reduced the field to the $4.3 \mu \mathrm{T}$ level, and seven visits later (visit 269) the animal began another criterion string $(6 / 6)$. In the final 19 visits of this animal with the field set at $1.3 \mu \mathrm{T}$, it failed to reach criterion and suddenly stopped coming to the feeder. Hence, the best field level discriminated by this animal was $4.3 \mu \mathrm{T}$.

Summary data for all of the animals tested in these experiments are shown in Fig. 3, plotted as the fraction of honeybees able to discriminate the magnetic field strength 


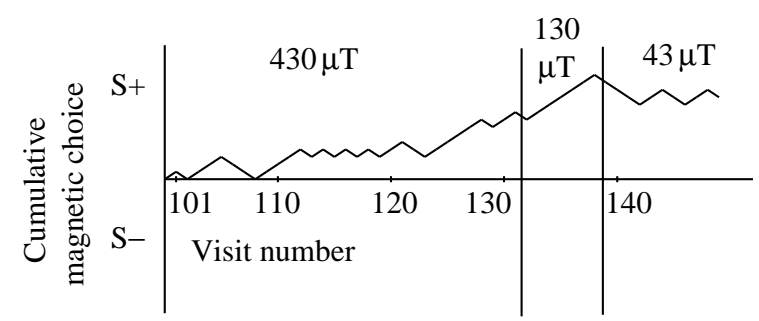

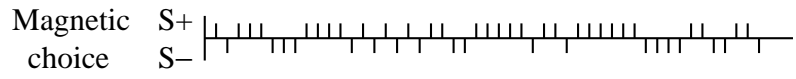

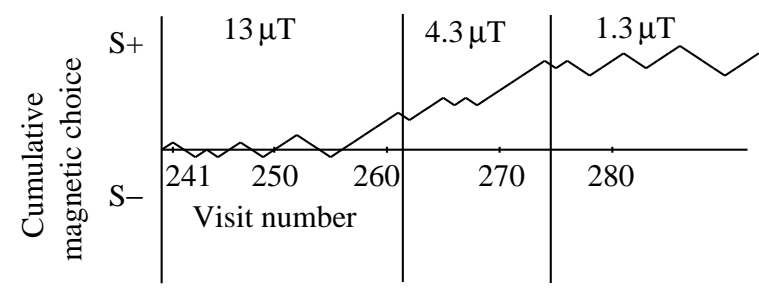

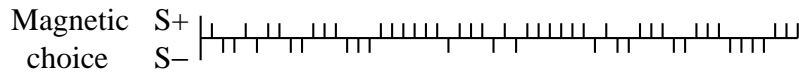

Fig. 2. Example data for a honeybee displaying sensitivity at $10 \mathrm{~Hz}$ down to the $4.3 \mu \mathrm{T}$ level in this conditioning experiment. The current and field strength were regulated by switching resistors in series from an initial starting value of $10 \Omega, 30 \Omega, 100 \Omega, 300 \Omega$, etc., which resulted in root-mean-squared (r.m.s.) field levels at the tunnel opening of $1300 \mu \mathrm{T}, 430 \mu \mathrm{T}, 130 \mu \mathrm{T}, 43 \mu \mathrm{T}$, etc. The horizontal tick bar shows the choice of the honeybee at each visit; $\mathrm{S}+$ indicates that the target associated with the magnetic anomaly was chosen (and the bee was rewarded immediately), whereas $\mathrm{S}$ - indicates that the target without the anomaly was selected (and the bee was punished). Cumulative response curves are shown for these data strings, along with the a.c. field levels used. Note that the results shown here are incomplete, but the main criterion strings are shown. A full description of these data is given as an example in the text. Vertical lines indicate the time at which the field levels were changed.

indicated. Data for the d.c. field experiments from Walker and Bitterman (1989b) are also shown for comparison, but are replotted so that the ordinate is in units of field strength at the landing platform rather than current in the coils. At $60 \mathrm{~Hz}$, only approximately $65 \%$ of the bees managed to reach criterion performance for the strongest stimulus, with the best animal retaining discrimination performance at $430 \mu \mathrm{T}$. This animal was unable to discriminate at $43 \mu \mathrm{T}$. In contrast, at $10 \mathrm{~Hz}$, the honeybees performed better, with over $30 \%$ discriminating down to the $130 \mu \mathrm{T}$ level, and the best individual conditioning at $1.3 \mu \mathrm{T}$. For comparison, the static geomagnetic field strength in Pasadena is approximately $45 \mu \mathrm{T}$.

\section{Discussion}

The results from this study demonstrate that the magnetic sensory system of honeybees operates best at extremely low a.c. frequencies of $10 \mathrm{~Hz}$ and below. The minimum field discriminated at $60 \mathrm{~Hz}(430 \mu \mathrm{T})$ is over four orders of

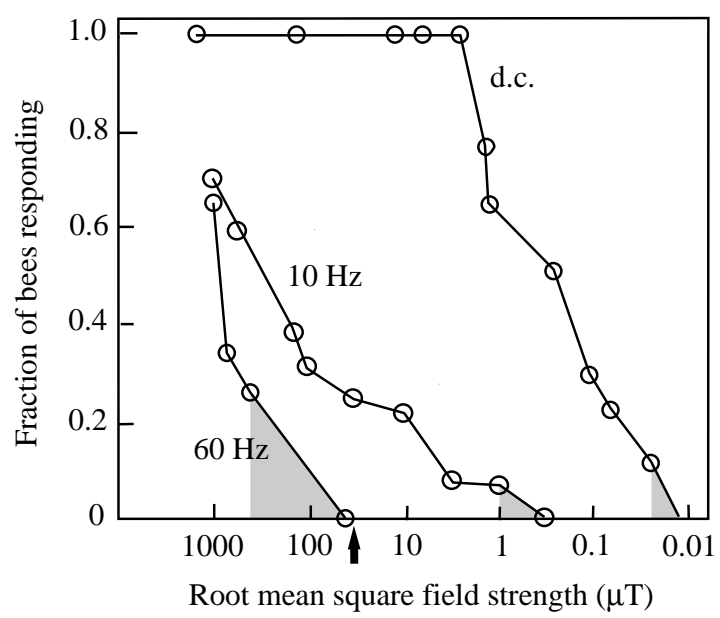

Fig. 3. Behavioral measurements of the ability of honeybees to discriminate extremely low-frequency a.c. magnetic fields. This figure shows the proportion of honeybees able to discriminate the given field stimulus. The data for nine honeybees exposed to the d.c. field are from Walker and Bitterman (1989b), and those from 11 honeybees tested at $60 \mathrm{~Hz}$ a.c. and 15 honeybees at $10 \mathrm{~Hz}$ a.c. are from the present study. Shaded areas at the lower end of each curve show the area of uncertainty between the performance of the best animal at that frequency and the subsequent level at which the animal did not discriminate. For comparison, the black arrow indicates the approximate magnitude of the d.c. geomagnetic field in Pasadena $(45 \mu \mathrm{T})$. The honeybee magnetoreception system is tuned for maximum sensitivities below $10 \mathrm{~Hz}$.

magnitude higher than the d.c. measurements of Walker and Bitterman $(1989 b, 26 \mathrm{nT})$, with the $10 \mathrm{~Hz}$ a.c. data giving intermediate sensitivity. The threshold level at $60 \mathrm{~Hz}$ is high enough to make it unlikely that the electromagnetic noise in the environment (generally less than approximately $1 \mu \mathrm{T}$, e.g. that produced by electrical power lines) could be detected except at exceedingly close range. Monte-Carlo simulations which mimic the entire experimental process (beginning with 1000 bees randomly guessing for up to 80 trials, and advancing to the next step if, and only if, they reach criterion performance) suggest that our results at $60 \mathrm{~Hz}$ are very close to the random chance level. Hence, if other organisms (such as humans) employ a magnetic sensory system similar to that of honeybees, it is unlikely that magnetoreception per se could produce the effects of weak 50 or $60 \mathrm{~Hz}$ magnetic fields.

These results are also compatible with the biophysical theory of magnetite-based magnetoreceptors (e.g. Gould et al. 1978; Kirschvink and Gould, 1981; Kirschvink et al. 1992). If biologically precipitated magnetite is the transducer of the magnetic sensory system in insects, as has been argued extensively on the basis of both magnetic (Gould et al. 1978) and behavioral (e.g. Walker and Bitterman 1989a,b; Kirschvink and Kobayashi-Kirschvink, 1991) data, then a frequency-dependence of the response could be a consequence of viscous damping of the motion of the magnetite (Kirschvink et al. 1992). In particular, the shape of the magnetite particle will affect its viscous damping properties, as the surface area 
and drag coefficients for elongate magnetosome chains are much larger than for a sphere of similar volume. Hence, the reduction in sensitivity with increasing frequency displayed by honeybees could be due to the magnetoreceptors having elongate chains of magnetite crystals, not unlike those present in magnetotactic bacteria (Blakemore, 1982) and fish (Mann et al. 1988). However, we cannot yet rule out the possibility that this reduction in sensitivity with increasing frequency has its basis in the neural filtering of the signal downstream from the actual magnetoreceptors. From an evolutionary perspective, honeybees were not exposed to a.c. magnetic fields above $10 \mathrm{~Hz}$ prior to human electrification of the planet (Skiles, 1985). As the insect nervous system was therefore not exposed to higher-frequency magnetic noise, there is no reason why neurological filtering mechanisms would have evolved. Direct electrophysiological recording from the actual receptor cells could, in theory, distinguish between these possibilities.

Although a competing hypothesis of magnetoreception which involves the optical excitation of electrons (Leask, 1977) makes no prediction concerning threshold sensitivity to magnetic fields in the extremely low frequency range $(0-60 \mathrm{~Hz})$, our data do not rule out this mechanism a priori. A neural filter, if it exists, might yield a reduction in sensitivity with increasing frequency of the sort reported here. However, several factors suggest that this is unlikely in honeybees. First, several experiments indicate that light is not necessary for magnetoreception (Lindauer, 1977; Kirschvink and Kobayashi-Kirschvink, 1991). Second, honeybee behavior in T-maze experiments is affected by a brief magnetic pulse (Kirschvink and Kobayashi-Kirschvink, 1991), which is a unique ferromagnetic effect (Kalmijn and Blakemore, 1978). Similar pulse-remagnetization experiments have recently demonstrated that ferromagnetic material (probably magnetite) is an essential component of the avian magnetoreceptor system (Wiltschko et al. 1994; Beason et al. 1995; Wiltschko and Wiltschko, 1995). Third, tiny magnets glued to the honeybee abdomen near the region of known magnetite concentration in the anterior dorsal abdomen interfere with magnetic discrimination in choice experiments (Walker and Bitterman, 1989a). As the magnetic field produced by these magnets decreases rapidly with distance, these results argue against the visual system being the site of the receptors. Finally, we note that the light-induced behavioral changes in magnetic compass orientation responses of Drosophila melanogaster (Phillips and Sayeed, 1993), of the newt Notophthalmus viridescens (Phillips and Borland, 1994) and of the European robin Erithacus rubecula (Wiltschko and Wiltschko, 1995) are quite different from each other. As cues from the visual system often have profound effects on all aspects of behavior, it is not surprising to find that visual cues might affect an animal's magnetic compass response. Hence, these effects do not require the presence of a light-dependent biomagnetic transduction system.

We thank Dr M. M. Walker and Professor M. E. Bitterman for their advice and suggestions for the replication of their threshold conditioning experiments. This work was sponsored by EPRI contract WO4307-03 and is contribution 5788 from the Division of Geological and Planetary Sciences of the California Institute of Technology.

\section{References}

Beason, R. C., Dussourd, N. and Deutschlander, M. E. (1995). Behavioural evidence for the use of magnetic material in magnetoreception by a migratory bird. J. exp. Biol. 198, 141-146.

BlaKemore, R. (1982). Magnetotactic bacteria. A. Rev. Microbiol. 36, 217-238.

Gould, J. L., Kirschvink, J. L. And DefFeyes, K. S. (1978). Bees have magnetic remanence. Science 201, 1026-1028.

KalmiJn, A. J. AND BlaKemore, R. P. (1978). The magnetic behavior of mud bacteria. In Animal Migration, Navigation and Homing (ed. K. Schmidt-Koenig and W. T. Keeton), pp. 354-355. Berlin: Springer-Verlag.

KIRSCHVINK, J. L. (1992). Uniform magnetic fields and doublewrapped coil systems: Improved techniques for the design of biomagnetic experiments. Bioelectromagnetics 13, 401-411.

KIRSCHVINK, J. L. AND GoulD, J. L. (1981). Biogenic magnetite as a basis for magnetic field sensitivity in animals. BioSystems 13, 181-201.

KiRschVInK, J. L. AND KobAYASHI-KIRSCHVINK, A. (1991). Is geomagnetic sensitivity real? Replication of the Walker-Bitterman conditioning experiment in honeybees. Am. Zool. 31, 169-185.

Kirschvink, J. L., Kuwajima, T., Ueno, S., Kirschvink, S. J., DiaZRicci, J. C., Morales, A., Barwig, S. AND Quinn, K. (1992). Discrimination of low-frequency magnetic fields by honeybees: Biophysics and experimental tests. In Sensory Transduction (ed. D. P. Corey and S. D. Roper), pp. 225-240. Society of General Physiologists, 45th Annual Symposium. Rockefeller University Press: New York.

LEASK, M. J. M. (1977). A physiochemical mechanism for magnetic field detection by migratory birds and homing pigeons. Nature $\mathbf{2 6 7}$, 144.

LiNDAUER, M. (1977). Recent advances in the orientation and learning of honeybees. Proc. XV Int. Congr. Ent. pp. 450-460.

Mann, S., Sparks, N. H. C., Walker, M. M. AND Kirschvink, J. L. (1988). Ultrastructure, morphology and organization of biogenic magnetite from sockeye salmon, Oncorhynchus nerka: implications for magnetoreception. J. exp. Biol. 140, 35-49.

Phillips, J. B. AND Borland, S. C. (1994). Use of a specialized magnetoreception system for homing by the eastern red-spotted newt Notophthalmus viridescens. J. exp. Biol. 188, 275-291.

PhILliPS, J. B. AND SAYEED, O. (1993). Wavelength-dependent effects of light on magnetic compass orientation in Drosophila melanogaster. J. comp. Physiol. A 172, 303-308.

SKILES, D. D. (1985). The geomagnetic field: its nature, history and biological relevance. In Magnetite Biomineralization and Magnetoreception in Organsims: A New Biomagnetism (ed. J. L. Kirschvink, D. S. Jones and B. J. MacFadden), pp. 43-102. New York: Plenum Press.

Walker, M. M. AND Bitterman, M. E. (1985). Conditioned responding to magnetic fields by honeybees. J. comp. Physiol. A 157, 67-73.

WALKER, M. M. AND BitTERMAN, M. E. (1989a). Attached magnets impair magnetic field discrimination by honeybees. J. exp. Biol. 141, 447-451. 


\section{J. L. KIRSCHVINK AND OTHERS}

WAlKer, M. M. AND BitTERMAn, M. E. (1989b). Honeybees can be trained to respond to very small changes in geomagnetic field intensity. J. exp. Biol. 145, 489-494.

Wiltschko, W., Munro, U., Beason, R. C., Ford, H. And WiltschKo, R. (1994). A magnetic pulse leads to a temporary deflection in the orientation of migratory birds. Experientia 50, 697-700.

WiLTSChKo, W. AND WILTSChKo, R. (1995). Migratory orientation of European robins is affected by the wavelength of light as well as by a magnetic pulse. J. comp. Physiol. A 177, 363-369. 